\title{
Ecología política y represas: elementos para el análisis del Proyecto HidroAysén en la Patagonia chilena ${ }^{1}$
}

\author{
Hugo Romero Toledo
}

\begin{abstract}
RESUMEN
Este artículo presenta una revisión crítica de estudios sobre represas y su posible articulación con los análisis de la ecología política. El objetivo es contribuir a la investigación sobre los alcances e impactos de estas megainversiones, entendidas como configuraciones socioecológicas que representan material y simbólicamente el poder de las elites. Sin embargo, la construcción de represas requiere la consolidación de discursos hegemónicos para realizar grandes transformaciones ambientales y sociales con respaldo ciudadano. A partir de esta argumentación se analiza el caso de HidroAysén en Chile, enfocado en los discursos que respaldan y justifican al proyecto desde las compañías eléctricas y el gobierno. Finalmente, se exploran las consecuencias que HidroAysén está teniendo en términos del conflicto ambiental, la tensión entre impulsores y opositores a las represas sobre la idea de Patagonia, y la promoción de cambios en la política energética.
\end{abstract}

Palabras clave: Ecología política, represas, discursos, HidroAysén, Patagonia chilena.

\begin{abstract}
This article presents a critical review of studies on dams and their possible articulation with an analysis of the political ecology. The aim is to contribute to the research on the reach and impacts of these mega-investments, which are understood as socio-ecological configurations that represent the power of the elite, both materially and symbolically. However, the construction of dams requires the consolidation of hegemonic discourses to make environmental and social transformations possible with citizen support. Given this argument, I analysed the case of HidroAysén in Chile, focusing on the discourses the energy companies and the government bodies that support this project. Finally, I explore the consequences that HidroAysén is having in terms of the environmental conflict; the tension between dam's promoters and opponents as to idea of Patagonia, and how changes to energy policy are promoted.
\end{abstract}

Key words: Political Ecology, dams, discourses, HidroAysén, Chilean Patagonia.

1 El autor agradece los comentarios de Dr. Gavin Bridge y Dr. Anthony Bebbington. De forma especial, se agradece a la Royal Geographical Society y Dudley Stamp Memorial por colaborar en el financiamiento de esta investigación, y a Becas-Chile por el financiamiento del programa de Doctorado en la Universidad de Manchester, Inglaterra. Proyecto FONDAP
№ 15130009 "The Centre for Social Conflict and Cohesion Studies (COES), Línea Geografías del conflicto". Artículo recibido el 5 de junio de 2012, aceptado el 11 de junio de 2013 y corregido el 2 de septiembre de 2013.

2 Escuela de Sociología, Universidad Diego Portales (Chile).E-mail: hugo.romero@mail.udp.cl 
La ecología política combina la preocupación por el cambio medioambiental desde la economía política, centrada en estudiar la dialéctica entre la sociedad y los recursos naturales, como también entre clases y grupos sociales (Blaikie, 1985; Blaikie \& Brookfield, 1987). Los enfoques de la ecología política pueden ser divididos en dos grandes grupos (Forsyth, 2003): el primero, derivado de enfoques estructuralistas sobre la degradación de la tierra producto de estados capitalistas $y / u$ opresores, y sus efectos sobre la población local y el ambiente. Esta ecología política "estructuralista" se ha concentrado en analizar los problemas de acceso a recursos, el ejercicio del poder económico, la coerción, la violencia, las políticas estatales, instituciones y los arreglos legales sobre el medio ambiente, relacionados con clases y estratificación social (Blaikie \& Springate-Baginski, 2007).

Un segundo enfoque conocido como "postestructuralista" ha estado centrado en la influencia de la historia y la cultura en conceptos y representaciones para entender el cambio medioambiental más allá de explicaciones económicas (Escobar, 1999). La tensión social sobre el medio ambiente estaría basada en la existencia de una pluralidad de formas de identidad, resistencia y subversión (Murdoch, 2006) desde donde se entienden las relaciones de poder, significados, actores, prácticas sociales, experiencias y cotidianidad de grupos sociales con medio ambientes determinados (Peet \& Watts, 1996; Peet et al., 2011; Sullivan \& Stott, 2000).

En el presente artículo se desarrolla un enfoque híbrido sobre ecología política. Por un lado, se recoge la existencia de condiciones político-económicas desde donde se realizan grandes transformaciones sociales y ambientales -como lo son la construcción de represas-, pero al mismo tiempo, se incorpora un análisis de discursos, desde donde se entienden las construcciones sociales y representaciones culturales que legitiman dichas transformaciones.

Como otros recursos naturales, el agua puede ser movilizada para generar riquezas. Esto depende de la distribución del poder en la sociedad el control y propiedad de la tierra, pero además el mismo régimen natural de agua. Es decir, la distribución de poder tiene estrecha relación con la cantidad, calidad, estacionalidad, variabilidad y disponibilidad de agua. Una de las formas más intensivas y extensivas de movilizar el agua es a través de la construcción de represas (Molle, 2005). De esta manera, la construcción de estas obras de infraestructura es un fenómeno complejo, que está en la intersección entre crecimiento económico, desarrollo, soberanía y territorio, y genera una tensión entre nativos y no nativos sobre el medio ambiente, el paisaje y el uso de recursos. En otros términos, la construcción de represas es un proceso cultural, político, judicial, social, económico y ambiental que trasciende el contexto inmediato de un proyecto hidroeléctrico determinado.

Desde la ecología política se ha planteado que la construcción de represas puede localizarse en el proceso de urbanización de la naturaleza para reproducir el metabolismo de la ciudades en el desarrollo de la modernización (Kaika, 2006). Dicho metabolismo crea medio ambientes en parte sociales y en parte naturales que contienen procesos químicos, físicos, sociales, económicos, políticos y culturales que son inseparables y altamente contradictorios (Swyngedouw, 2003). La geografía crítica marxista denomina a este fenómeno "producción de naturaleza", es decir, la creación humana de un medio ambiente intervenido, por ejemplo a través de paisajes diseñados e incluso organismos genéticamente modificados. Esta "naturaleza" sería natural y al mismo tiempo social, y su producción masiva está estrechamente relacionada con la expansión del sistema capitalista (Smith, 1984; Braun \& Castree, 1998; Swyngedouw, 1999). La producción de estos medio ambientes socionaturales no es social ni políticamente neutra, y expresa relaciones de poderes física, sociales, culturales y económicas, relacionadas con escalas sociopolíticas nacionales e internacionales, que representan la visión de la elite sobre el espacio (Harvey, 1996; Swyngedouw, 2007; Bakker, 1999).

Las represas serían un ejemplo de estos medio ambientes "socionaturales". Ellas sirven para manejar racionalmente el agua, mejorar el transporte fluvial y para la producción de electricidad, dentro de un proceso general de transformación de toda la naturaleza en una mercancía (Bakker, 1999). Esta trasforma- 
ción socionatural es posible a través del proceso de producción, que relaciona procesos materiales y simbólicos de representación de la naturaleza (Swyngedouw, 2003). Este argumento puede ser trazado desde los trabajos de Steward y Wittfogel sobre las "sociedades hidráulicas" en el mundo antiguo como Mesopotamia, Egipto, India y China, cuyas elites formadas por científicos, ingenieros, sacerdotes y burocracias agroempresariales, modificaron los ríos a través de represas y canales, reorganizando el trabajo, maximizando la producción agrícola y concentrando hegemonía (Worster, 1982).

En términos político-ecológicos, las represas han sido ocupadas como un símbolo de progreso tecnológico que refleja la victoria del hombre sobre la naturaleza (Kaika, 2006; Nüsser, 2003). En este proceso, el sistema político depende de la producción exitosa de nuevos socioambientes para sustentar el creciente metabolismo urbano, lo que significa que nuevas represas deben ser constantemente construidas. Algunos académicos dicen que la construcción de grandes represas es una actividad que solo puede ser abordada por gobiernos nacionales, no solamente por los altos costos de inversión involucrados, sino además porque solamente el Estado podría organizar la distribución de beneficios y los costos (Heming, et al., 2001: 208). En la mayoría de los casos, el Estado juega un rol importante en los procesos de propuesta de proyectos, construcción, negociación, mitigación y relocalización de las comunidades afectadas.

El desarrollo hidroeléctrico casi siempre requiere el involucramiento de capital y la experiencia transnacional, dada la escala de la construcción e impactos que generan (Bakker, 1999). En esta línea aparecen consultoras multinacionales de ingeniería y construcción como International Commission of Large Dams (ICOLD). En varios de los casos analizados en el Tercer Mundo, los principales inversionistas en represas son compañías de EE.UU., Canadá, España y Noruega. Al mismo tiempo, los proyectos hidroeléctricos son también financiados por instituciones internacionales como el Banco Mundial (por ejemplo en los proyectos Lesotho Highland Water Project y Three Gorge Dam en China), Association of Southeast Asian Nations
(ASEAN) y Asia-Pacific Economic Cooperation (APEC); junto con bancos multilaterales de desarrollo de África, Asia y América Latina. De la misma manera, agencias de crédito de países del Primer Mundo están detrás de la mayoría de los proyectos de grandes represas en el mundo.

La mayoría de estas intervenciones socionaturales cuenta con una oposición organizada, donde confluyen el movimiento mundial antirrepresas y redes internacionales de defensa del medio ambiente y los derechos humanos como: International Rivers Network, Natural Resources Defence Council, Forestethics, Free Flowing Rivers, Greenpeace, entre otras. Dichas ONGs se vinculan con agencias nacionales de justicia social como movimientos sociales y partidos políticos, generalmente de izquierda y verdes. Además confluyen organizaciones de la sociedad civil y ONGs locales vinculadas no solamente a temáticas ambientales, sino también a la conservación del patrimonio cultural. Por último, y en especial para el caso de América Latina, la Iglesia Católica es un actor relevante en la defensa de las comunidades afectadas, como también en el proceso de negociación con el gobierno y las empresas.

Como proyecto ecológico-político las represas representan un nuevo momento en el cual la imagen del país debe ser imaginada y materialmente construida: Postguerra (EE. UU., ex URSS, Grecia, Japón, entre otros), Postguerra Civil (España), Postcolonialismo (Lesotho, India), Postguerra Fría (Lao, Cambodia, China), Postdictaduras (Brasil, Chile). Por ejemplo, la construcción de represas en el Amazonas han servido no solamente por la necesidad de energía, sino también para que la elite económica y política se "sacudan la imagen de un país dominado por bosques e indios" (Cummings, 1995: 153).

Este proceso de cambio ecológico-político, a través de la construcción de nuevos medio ambientes para insertarlos dentro de circuitos económicos, es profundamente injusto social y ecológicamente, antidemocrático e incluso racista con las comunidades locales, las cuales son alienados de sus tierras, de sus formas de vida, rituales, manifestaciones culturales y producción económica. La población afectada casi siempre 
son indígenas: Sami en Noruega; Cree y Inuit en Canadá; Waimiri-Atroari en la Amazonía; Mapuche-Pehuenche en Chile, entre otros casos; además de comunidades de campesinos agrícolas, ganaderos, pescadores, leñadores. En todos los casos son gente pobre, aislada, tradicional y no totalmente incorporada a las dinámicas del capitalismo. Por estas razones, la resistencia a grandes represas es frecuentemente contra el Estado (Martínez-Alier, 1998), dado que el Estado capitalista moderno necesita expropiar el valor de la tierra para la elite minoritaria nacional o extranjera (Cummings, 1995: 159).

En algunos casos, como el de los Sami contra el Estado noruego, el conflicto sobre represas contribuyó a un incremento del reconocimiento legal de los derechos de los indígenas en los países escandinavos, en términos de reforma administrativa, y la promesa de cambios constitucionales, legales y políticos (Howitt, 2001). Lamentablemente, en la mayoría de los otros casos, las personas y comunidades son forzadas a migrar y a continuar marginadas, como el caso de los Pehuenche en el Alto Biobío.

\section{El debate sobre las represas}

Mucha de la literatura existente atribuye la construcción de represas al crecimiento de la población y el aumento de la actividad económica, generado por el incremento de la demanda humana sobre el agua y sus servicios relacionados. El crecimiento económico, el cambio tecnológico, la distribución del ingreso, y el estilo de vida globalizado asociado a la urbe y el consumo de bienes y servicios, afectan el nivel de la demanda de agua que pasa a ser un bien escaso y desigualmente distribuido en el tiempo y el espacio (Altinbilek, 2002).

El discurso de las compañías constructoras de represas básicamente apuntan a que: a) la hidroelectricidad es limpia, b) el agua que fluye libre hacia el océano es desperdiciada, y c) los residentes locales se benefician del proceso de construcción y puesta en marcha de estas megainversiones (Rosenberg et al., 1995). Al respecto, las represas hidroeléctricas tienen una lista de características positivas que explican el fuerte apoyo y promoción por parte de gobiernos y empresas. Algunos plantean que las represas son altamente eficientes en la producción de energía eléctrica en comparación con otras fuentes como fósiles, nucleares y geotérmicas en cuanto a su costo, complejidad estructural y construcción (Erakhtin, 1998). Entre sus ventajas significativas están que las represas pueden ser usadas en el peak de sus operaciones y que ocupan al agua como un recurso renovable y doméstico, por lo cual usualmente los gobiernos del Tercer Mundo las prefieren por sobre los combustibles fósiles que deben importar. Adicionalmente el agua para la producción hidroeléctrica tiene un uso no consuntivo y es considerado no contaminante (Altinbilek, 2002). Junto con la generación de energía, las ventajas de las represas se asocian a la protección de inundaciones, regulación de las corrientes y usos múltiples como: atracción turística, desarrollo de diversos tipo de agricultura y como soporte a la urbanización e industrialización por el incremento de la concentración de la población (JCLD, 2009).

Por su parte, los discursos en contra de las represas plantean que su producción no es limpia debido a que se destruye el paisaje a través de inundación de vastas áreas de bosques en la formación de las reservas, se desecan los cuerpos de agua debido a la desviación del agua, se genera la erosión de la costa causada por el embalse del lago y se desvían las aguas a través de canales artificiales desde los ríos existentes afectando su capacidad hidráulica. Además, emiten gases de efecto invernadero causados por la inundación de los bosques de tierras altas y la turba de zonas terrestres, y la posible contaminación por mercurio que afectaría directamente a los peces y a los animales, y a las personas que los consumen (Rosenberg et al., 1995).

La escala de las grandes represas genera una falta de certezas sobre sus riesgos, debido a la manipulación de sistemas de soporte de vida como lo son las cuencas de los ríos, y la falta de capacidad gubernamental para lidiar con las complejidades de grandes proyectos de infraestructura (Scudder, 2005). Los impactos de las represas son irreversibles en los componentes naturales y humanos de los medio ambientes, especialmente para aquellos que dependen directamente de los flujos naturales de los ríos. Ejemplos de todo el mundo muestran una estrecha relación en- 
tre impactos biofísicos y sociales debido a la transformación del paisaje y los ciclos hidrológicos. Simultáneamente, las economías de subsistencia son sumamente sensibles y vulnerables frente al desarrollo industrial debido al cambio en el uso y manejo de recursos y la organización de la producción y distribución.

El río es un recurso crítico para las formas de vida de los residentes de las cuencas, es decir, las personas que usan el río para pesca, irrigación, como agua para beber y medio de transporte; y para aquellos que consideran al río como un elemento central en su cosmovisión, principalmente comunidades indígenas y campesinos. Generalmente, la energía producida no es para consumo local, sino para las necesidades industriales de exportación. Debido a esto, todos los usos no industriales del ríos, es decir, el uso de sus recursos y la construcción material y simbólica del medio ambiente, son virtualmente invisibles para el modelo hegemónico (Howit, 2001).

Los ríos son elementos centrales en muchos paisajes como corredores naturales de energía, materia, especies y biodiversidad. Sus ecosistemas están formados por elementos bióticos y abióticos, que incluyen no solamente sus habitantes acuáticos en los canales, sino también desde las capturas de agua hasta el mar, con todo el sistema de aguas subterráneas, sectores naturales de inundación, estuarios y ecosistemas que dependen de inputs de agua fresca. En este sentido, el concepto de el río como un "continuo" (McCartney, 2009) debería ser central en la evaluación de represas, considerando el rol de los nutrientes y sedimentos, su relación con los organismos que habitan y viven del río, y los ciclos por los cuales atraviesan con sus variaciones, profundidades y patrones de flujo espacial y temporal, que son responsables de la diversidad de hábitats, y por lo tanto, de la diversidad ecológica y social.

\section{Los impactos de las represas}

Ha habido reconocimiento de parte de todos los sectores vinculados a las represas que sus impactos son complejos y pueden ser de gran alcance. Sin embargo, los impactos de cada represas son únicos (McCartney, 2009). La literatura menciona que si bien hay impac- tos que ocurren durante la construcción de las represas, usualmente los más importante se dan en el largo plazo una vez que esta entra en operación (Ledec \& Quintero, 2003). Además, generalmente las represas hidroeléctricas posibilitan el desarrollo de nuevos proyectos de inversión asociados a la expansión urbana, facilidades industriales, y nuevas suministros de agua.

Los impactos sociales pueden ser:

- positivos (por ejemplo el mejoramiento del bienestar resultante de nuevos accesos a irrigación), o negativos (como la relocalización de personas, reducción de la pesca por el control de la corriente).

- directos (asociado a traumas culturales por relocalización involuntaria), o el resultado de una cascada donde impactos ambientales generan impactos económicos y ellos se transforman en la causa de impactos sociales.

- también pueden ser locales, es decir, concentrados en el lugar de la represa, o nacionales, porque ocurren en lugares lejanos a la misma, donde el agua o la electricidad son consumidas (Adams, 2000:1).

Dada la revisión de la literatura, la construcción de represas es un factor crucial en las transformaciones intra e interculturales. Su construcción genera cambios en las estructuras económicas y políticas, debido a la forma en que la lógica del mercado penetra a territorios tradicionalmente excluidos a través de la comodificación del paisaje del río, la escala de la represa y los cambios en el régimen del flujo del río. De esta manera se intervienen procesos ecológicos-culturales en el que el sistema de manejo de recursos es movilizado social, ecológica, locacional y temporalmente. Más aún, afecta la sobrevivencia, los derechos, el reconocimiento, la autodeterminación y el autogobierno en el uso de la tierra y el agua que empiezan a operar bajo la lógica de las represas (Howitt, 2001). Se alteran significativamente los lazos sociales en las villas de afectados, y cambia la relación con la tierra, las actividades económicas y el sentido que ellas tenían para las comunidades locales (Tilt et al., 2009).

Los problemas generados por este tipo de proyectos son importantes y complejos. 
Desde el punto de vista social ellos incluyen la preservación del patrimonio cultural, la relocalización de personas, una compensación justa por los bienes perdidos, la creación de nuevas comunidades, la salud y el bienestar de las poblaciones afectadas tanto aguas arriba como aguas abajo; la supervivencia económica y el desarrollo de estas poblaciones a largo plazo, temáticas de género y derechos de las minorías (Égré \& Senécal, 2003: 215).

La relocalización es considerada la más severa forma de impacto social generada por las represas (Égré \& Senécal, 2003). La mayoría de los relocalizados se ha empobrecido en relación a la situación anterior de la construcción, debido la impotencia derivada de la pérdida de control sobre los recursos, pérdida de poder político, y más dramáticamente, pérdida sobre el poder de tomar decisiones sobre el cómo y dónde vivir (Scudder, 2005: 23). El centro de las medidas de reconstrucción de las formas de vida es la compensación monetaria de parte de las instituciones $\mathrm{u}$ organizaciones asociadas al proyecto (JCLD, 2009). Sin embargo, la mayoría de los relocalizados sufren perturbaciones en sus patrones de asentamiento en el proceso forzado de "modernización" en las nuevas villas, precisamente por la ruptura de relaciones basadas en la familia y el cambio en el acceso a recursos (principalmente pesca y caza). El sedentarismo, la llegada de patrones culturales occidentales, sin una forma de sustentarlo debido al desempleo, resultan en una fuente de estrés constante para las comunidades (Howitt, 2001).

En la literatura sobre migración "involuntaria" o "forzada" existe una categoría especial para aquellas personas que son desplazadas por proyectos de infraestructura como las represas. Dichas personas son desplazadas por consecuencia del "desarrollo" (Heming et al., 2001). Como los refugiados políticos y ambientales, las personas desplazadas por represas deben dejar sus hogares y sus medios de producción, y deben migrar contra su voluntad. Pero en contraste con los refugiados políticos y ambientales, la migración por represas es un proceso planeado por el gobierno. Esto significa que el Estado debe tomar la responsabilidad de relocalizar y restablecer las formas de vida de los desplazados. La gente desplazada por represas normalmente no deja sus países de origen y son relocalizados internamente, tienen tiempo para hacer preparativos para su reasentamiento, e incluso pueden tener la opción de elegir un lugar, principalmente bajo planes del gobierno. Debido al alto costo que significa el traslado de personas se espera que estos mejoren material y socialmente sus condiciones de vida en comparación a cómo estaban antes de ser movidos (Heming et al., 2001), y dado que no tendrán la oportunidad de volver a sus lugares de origen, aun si el proyecto hidroeléctrico termina su vida útil. La mayoría de estos elementos ha estado ausente en el reciente debate sobre la construcción de represas, especialmente en los proyectos hidroeléctricos en la Patagonia.

Por ejemplo, en el caso de China, con una larga historia de construcción de represas, las consecuencias de la relocalización han tomado tres formas, según Heming et al. (2001):

- Empobrecimiento Económico: Disminución de las áreas de tierra per cápita y la degradación de la calidad de la tierra. Al mismo tiempo, entre los reasentados la indemnización se fijó en una tasa muy baja en comparación con los bienes perdidos y no reflejó la necesidad de construir una nueva vivienda familiar e invertir en la capacidad de producción para restaurar los estándares de vida. El desplazamiento y reasentamiento dejó a las personas en peor situación, con una alta incidencia de la falta de tierras, el desempleo y la falta de vivienda.

- Estabilidad Social: Varios conflictos dañaron la relación entre los relocalizados y con los nuevos vecinos. Las razones se asocian a la insatisfacción con los nuevos medio ambientes donde son insertados y con las nuevas condiciones de producción, lo que se traducen en menores ingresos y una pérdida de los estándares de vida, además de ser vistos como extranjeros por la nueva comunidad.

- Degradación Ambiental: Los cambios en el medio ambiente impactan las formas de vida de los desplazados y su conexión tradicional con la tierra. Además se genera una mala adaptación a las nuevas condiciones principalmente centradas en la necesidad de agua, comida y combustible, y los usos de laderas y bosques. 
Además, en algunos casos, la exposición a un nuevo estilo de vida, asociado a las urbes y a la cultura occidental, ha impactado fuertemente los patrones ecológico-culturales de las comunidades relocalizadas. En otros, el suministro de agua en las tierra de reasentamiento puede incluso transformarse en un problema de salud pública para aquellas personas acostumbradas a un río de flujos libres (Scudder, 2005). Al mismo tiempo, la relocalización conlleva la instalación de nueva infraestructura que raramente está asociada con la cultura de las comunidades reasentadas y con sus necesidades de empleo.

Los Cree en Canadá solamente consiguieron empleos mal pagados, de corta duración, y de bajo entrenamiento que afectaron incluso su economía existente (Rosenberg et al., 1995). Además, los grandes proyectos hidroeléctricos implican necesariamente la invasión de forasteros en los territorios tradicionales de la población indígena y campesinas, principalmente facilitado por las nuevas carreteras, aeropuertos y puertos. En el caso de los Cree, el sistema de tenencia de la tierra, la abundancia y distribución de los recursos pesqueros y la fauna silvestre, fueron interrumpidos por la invasión externa, con los consiguientes impactos sociales y ecológicos adversos.

Las grandes represas son generalmente justificadas por su beneficios macroeconómicos a escala regional y nacional, sin embargo, sus impactos físicos y simbólicos se concentran localmente (Altinbilek, 2002). A partir de los trabajos la Comisión Mundial de Represas (WCD, 2000), Comisión Japonesa de Grandes Represas (JCLD, 2009) y de los trabajos de Scudder (2005) y Dorcey (1997), he agrupado los impactos producidos por las represas en las siguientes categorías:

\section{Ecológicas:}

- Generalmente se afectan áreas de alta calidad medioambiental.

- Ciclos de agua (captación, piscinas, lagos, lagunas, pantanos, deltas, fiordos y costas).

- Ruptura de áreas de migración de animales.

- Fragmentación de ecosistemas del río.

- Modificación de los procesos de erosión y deposición.

- Inducción a la sismicidad.
Económicas:

- Inundación de terrenos productivos, bosques, territorios de caza y pesca

- Creación de nuevas villas y rápido crecimiento urbano

- Efectos sobre el mercado laboral local

- Apertura de la región al desarrollo industrial

\section{Sociales:}

- Cambios en la subsistencia y formas de vidas dependientes

- Desplazamiento de habitantes

- Incremento de la estratificación social

- Exclusión de las comunidades locales del proyecto y sus beneficios

\section{Culturales:}

- Cambios en las formas de vida

- Cambio en las cosmovisiones

- Perturbaciones sobre el patrimonio cultural

- Perturbaciones de rituales, ceremonias y sitios sagrados

- Alteración de los liderazgos locales

- Cambio en los roles de género

\section{Políticas:}

- Uso de la regulación del Estado contra el control de recursos por parte de campesinos e indígenas

- Disrupción de los mecanismos locales de participación, representación y negociación

- Creaciones de nuevas instituciones que entreguen servicios en las comunidades para los fines de los proyectos, ya sean públicas o privadas

- Cambios en las políticas sobre administración de la tierra, acceso a la tierra, explotación del bosque, exploración mineral, protección ambiental y desarrollo hidroeléctrico.

Sobre la salud de las personas:

- Proliferación de enfermedades (tales como malaria, schistosomiasis, bilharzias y onchocerciasis)

- Aumento de los casos de enfermedades de transmisión sexual

- Cambios en los patrones alimenticios y nutricionales

- Riesgo de sismicidad 
- Exposición a elementos peligrosos (mercurio)

Debido a que HidroAysén es hasta el momento solo un proyecto, sus efectos ecológicos, económicos, sociales, culturales y políticos aun no pueden ser evaluados. Al mismo tiempo, no existen estudios de represas en el estado de preconstrucción por lo que poco se sabe de los procesos anteriores a la masiva movilización de capital, personas y naturaleza. Mi propuesta es ocupar el enfoque "postestructuralista" de la ecología política para entender cómo discursos y representaciones son utilizados como ejercicio de poder para crear "verdades" y así facilitar la transformación social y ambiental.

\section{Método utilizado}

Para estudiar el caso del proyecto $\mathrm{Hi}$ droAysén, se han utilizado fuentes secundarias, principalmente textos oficiales de los gobiernos, discursos de autoridades y proponentes de represas, otros trabajos académicos, reportajes de medios de comunicación y textos producidos por las campañas opositoras a la construcción de represas. Los datos han sido analizados a través de Análisis Crítico de Discursos (Van Dijk, 1998) para analizar la importancia social y uso de discursos, declaraciones, textos, artefactos, espacios y lugares. De acuerdo a esta perspectiva, los discursos son actos lingüísticos y no lingüísticos que constituyen prácticas sociales. Usos particulares del lenguaje utilizan ciertas categorías que permiten observar cómo el poder, la dominación y las desigualdades son producidas, reproducidas y resistidas en contextos políticos y sociales (Van Dijk, 2001). En esta perspectiva los discursos están social e históricamente situados (Jørgensen \& Phillips, 2002), y circulan por la sociedad a través de medios oficiales, medios de comunicación de masas y redes sociales de diverso alcance desde donde actúan para generar consensos sobre "versiones particulares del mundo". El presente artículo analiza discursos desde una aproximación top-down (Fairclough, 1995, Woods, 2006), dado que el interés es conocer cómo se proponen las transformaciones sociales y ambientales principalmente desde el gobierno y las elites.

\section{La ecología política de la represas en Chile}

La expansión de la hidroelectricidad en Chile tiene directa relación con la aplicación del neoliberalismo autoritario, que significó cambios constitucionales y cambios jurídicos e institucionales sobre el manejo del agua, junto con la creación de un sector eléctrico desregulado (Bernstein, 1991; Bauer, 2002, 2009). De acuerdo a Luz Domper de "Libertad y Desarrollo" el $80 \%$ de la inversión hidroeléctrica se ha efectuado con el actual marco legal y político (Estrategia, 2010), lo que según Prieto \& Bauer (2012) grafica una clara preferencia por la producción hidroeléctrica del modelo neoliberal chileno.

Bajo el discurso de eficiencia y racionalización, se favoreció la inversión extranjera y la conformación de monopolios eléctricos que han concentrado los derechos de agua. El Estado pasó a tener un rol consultivo, permitiendo la existencia de distintos mercados eléctricos que operan con una institucional ad hoc (Budds, 2009, 2004). Tres grandes empresas se han fortalecido en este proceso controlando casi la totalidad de la generación de energía del Sistema Interconectado Central (SIC) que abastece de electricidad a más del 90\% de la población de Chile, y donde se desarrollan actividades industriales, de servicios y extractivas: ENDESA (68\%), Colbún (18\%) y GENER (5\%) (Prieto \& Bauer, 2012).

El discurso sobre la hidroelectricidad en Chile no es técnico, sino político. Se argumenta que las reservas de combustibles fósiles son pocas y no cuentan con la inversión necesaria (Bauer, 2002), que por tanto debe optarse por un tipo de combustible que permita la soberanía energética y la seguridad nacional (Tokman, 2008). De esta forma, se ha argumentado que el agua debe ser la fuente de energía de Chile, aun cuando el país es afectado por sequías principalmente generadas por el Fenómeno de la Niña. Esto ha significado la necesidad de asegurar la variabilidad hidrológica expandiendo la producción hidroeléctrica hacia aquellos territorios del país que cuenten con las "condiciones naturales" para asegurar la producción hidroeléctrica. En este escenario se plantean los proyectos hidroeléctricos en la Patagonia 
chilena, principalmente en Aysén, donde ENDESA es dueña del $98 \%$ de los derechos de agua actuales (Prieto \& Bauer, 2012: 137138).

\section{El proyecto HidroAysén}

El proyecto HidroAysén pretende construir cinco centrales hidroeléctricas (dos en el río Baker y tres en el río Pascua) en la región de Aysén, localizada en la Patagonia Occidental (ver Figura $\mathrm{N}^{\circ} 1$ ). El proyecto contempla la inundación de 5.910 hectáreas, que equivale al $0.05 \%$ de la Región de Aysén, para generar 2.750 MW que se integrarán al SIC, suficiente para iluminar una ciudad de cuatro millones quinientos mil habitantes. Creada el año 2006, HidroAysén es una sociedad anónima perteneciente en un $51 \%$ a ENDESA/ENTEL y en un $49 \%$ a la empresa chilena privada COLBÚN. Con una inversión solamente en los embalses que se estima en US\$3.200 millones, HidroAysén como consorcio de ENDESA y COLBÚN controlará el $80 \%$ del mercado. El traslado de la energía desde su generación en la Patagonia hasta los centros de consumo ha significado un debate sobre si HidroAysén deberá construir una de las líneas de transmisión más larga del mundo (2.200 kilómetros de largo) con un impacto ambiental y visual de proporciones (Rodrigo \& Orrego, 2007) o si el Estado chileno, después de 40 años de neoliberalismo, intervendrá construyendo una "carretera eléctrica públi$\mathrm{ca}^{\prime \prime}$ que sirva para conectar los proyectos en la Patagonia (HidroAysén y Energía Austral), como también proyectos de energía renovables no convencional al SIC, y su posible integración con el Sistema Interconectado del Norte Grande (SING).

En mayo de 2011, frente a la expectación de políticos, empresarios, ambientalistas y ciudadanos, el Proyecto HidroAysén fue aprobado por la Comisión de Evaluación Ambiental de Aysén. Las autoridades regionales de Aysén pusieron ciertas condiciones para que este se desarrolle. Las más importantes fueron: una auditoría ambiental independiente, bajar las tarifas de electricidad a la región de Aysén en un $50 \%$ y talar completamente la flora de los lugares que serán inundados para evitar emanaciones de $\mathrm{CO}_{2}$, ambas obras de un alto costo, a los cuales HidroAysén se ma- nifestó en contra (El Mercurio, 9 de mayo de 2011; La Tercera, 15 de mayo de 2011).

El rechazo ciudadano al Proyecto $\mathrm{Hi}$ droAysén alcanzó el 61\% antes de su aprobación (Encuesta IPSOS, abril de 2011), cifra que se elevó al $74 \%$ después de tomada la decisión (La Tercera, 15 de mayo de 2011). Durante mayo y junio de 2011 se realizaron jornadas de protestas en las principales ciudades de Chile, con cientos de miles de participantes a nivel nacional, lo que demostró que HidroAysén más que ser un conflicto ambiental era un tema sensible para los chilenos en lo que respecta a la Patagonia como territorio, y su patrimonio natural y cultural; la soberanía sobre los recursos naturales; las necesidades de democratización del sistema político y representatividad de las administraciones locales y regionales; y a la vinculación extrema que tienen en Chile los sistemas económico, político y judicial.

\section{Los discursos sobre HidroAysén}

El discurso de HidroAysén es que este proyecto estará "contribuyendo a la independencia energética de Chile al utilizar un recurso limpio, renovable y chileno, como es el agua" (www.hidroaysen.cl). Durante el 2011 una serie de comerciales de HidroAysén aparecieron por la televisión abierta con el mensaje "si Chile no duplica su energía desde hoy, en diez años más funcionará a medias", "con HidroAysén Chile tendrá la energía renovable que necesita", "el agua es energía limpia y no produce emisiones, y Chile tiene agua en Aysén", "el agua tiene la energía para Chile, y Chile tiene agua en Aysén".

El discurso de HidroAysén se sustenta en que es necesario agregar $12.000 \mathrm{MW}$ al sector eléctrico para sostener el ritmo del crecimiento económico chileno. Sin embargo, $\mathrm{Hi}$ droAysén solo aportará un $25 \%$, por lo que se hace necesario desarrollar todo tipo de proyectos eléctricos, incluyendo renovables no convencionales y nuclear si es que se quiere alcanzar el desarrollo. Un segundo elemento es que los ríos de la Patagonia reducirán de un $23 \%$ a un $9 \%$ la variabilidad hídrica debido a los deshielos durante el verano, pudiendo suplir la demanda del centro del país que se ve afectado por la sequía estacional 
Figura $\mathrm{N}^{\circ} 1$

Localización del proyecto HidroAysén

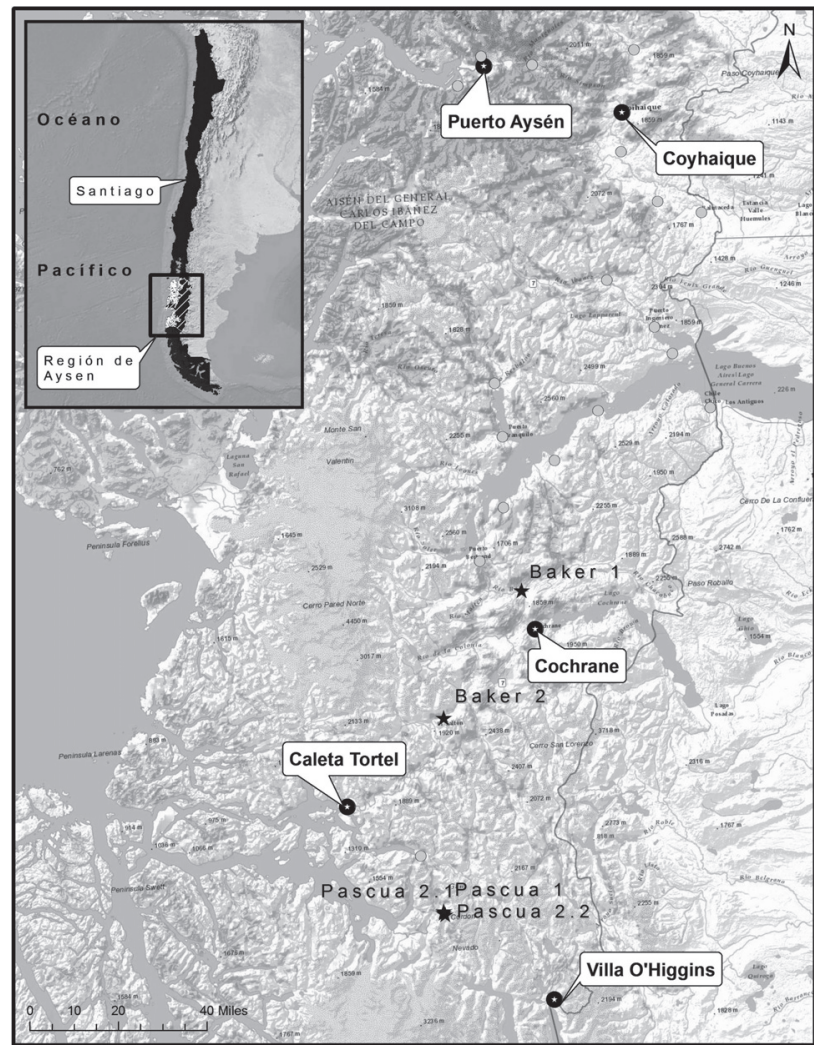

Fuente: Elaboración propia.

(Daniel Fernández, 2011). Al mismo tiempo, se prevén alteraciones en las precipitaciones en Chile producto del cambio climático, lo que hace necesario explotar aquellas áreas donde existen "condiciones naturales" como Aysén, donde el sistema de campos de hielo y grandes lagos son colapsados por el discurso económico-político, que entiende estas características geográficas como reservas hidroeléctricas: "el agua es el petróleo de Chile" (Rosenblut, presidente de ENDESA, 2012).

Sobre esta base que mezcla elementos técnicos con naturales, se desarrolla un tercer elemento discursivo que apela al nacionalismo y a la independencia energética; de esta manera HidroAysén se presenta a sí mismo como "un proyecto chileno" (Salazar, 2008), ya que pertenece en un $70 \%$ a Colbún, a las fondos de pensiones y a ENDESA (Fernández, 2011). Así se pretende reducir las críticas
Figura $\mathrm{N}^{\circ} 2$

Campaña de Hidroaysén.

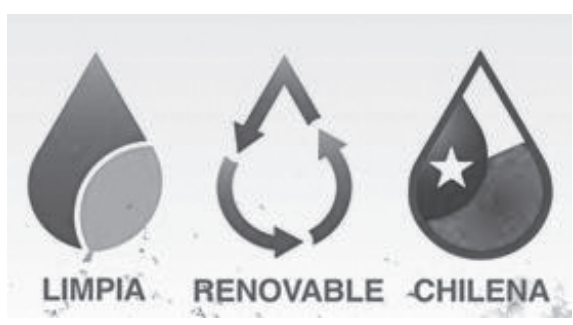

Fuente: HidroAysén, 2013.

hacia el rol del capital transnacional en la economía chilena, y la transformación de espacios geopolíticos sensibles como la $\mathrm{Pa}$ tagonia, y crear una identificación nacional con el proyecto: "HidroAysén es un proyecto país", que utilizaría al agua chilena como un 
recurso confiable que no depende de externalidad económicas (el alto valor del petróleo) y geopolíticas (la crisis del gas con Argentina).

Un cuarto elemento discursivo de $\mathrm{Hi}-$ droAysén es la protección ambiental. El proyecto se presenta como ambientalmente sustentable y renovable, ya que no afecta al agua como recurso primario. De esto se desprende que producir con agua es limpio y soberano, lo que lo hace al mismo tiempo altamente competitivo. HidroAysén significaría la sustitución de 16 millones de toneladas de $\mathrm{CO}_{2}$ por año si es que la misma cantidad de energía fuera producida por carbón. Al mismo tiempo, el agua es un recurso predictible por lo que entrega mayor seguridad que la energía solar o eólica. De esta manera, HidroAysén equivale a siete termoeléctricas o a cuatro plantas nucleares (Fernández, 2011).

Un quinto elemento discursivo es la eficiencia. HidroAysén promete ser más eficiente en la inundación de tierras y la producción de energía que otros grandes proyectos hidroeléctricos en América Latina y con cualquier otra fuente de energía, principalmente con la renovable no convencional donde se ocuparían hasta 100 mil hectáreas de generadores de viento; hasta 180 mil hectáreas de paneles solares o la construcción de 275 centrales de pasadas (www.hidroaysén.cl).

Por último, el proyecto HidroAysén promete desarrollo para la región de Aysén, que fue protagonista de intensas movilizaciones sociales durante el 2012, exigiendo mayor inversión pública, subsidios a los combustibles, un trato especial como zona extrema y mayor involucramiento en las decisiones sobre grandes proyectos de inversión. HidroAysén plantea mejorar la infraestructura de los caminos, puertos y aeropuertos, empleo y revitalización de la economía local, educación y entrenamiento para la fuerza laboral local, el apoyo a la comunidad local mediante proyectos para financiar emprendimientos, proyectos culturales y ambientales, y la reducción del precio de la electricidad que pagan las personas.

De esta manera HidroAysén se presenta a sí mismo como un proyecto que tiene la solución para la crisis energética, la crisis ambiental y la crisis económica, construyen- do un discurso hegemónico desde donde se plantea que "este proyecto no se plebiscita, este proyecto se aprueba si cumple con los requisitos de la ley y por los organismos técnicos (Daniel Fernández, 2011).

Dicho discurso está en directa concordancia con lo planteado por los últimos gobiernos de centro izquierda y centro derecha: la producción de energía, especialmente de hidroelectricidad, es un elemento clave para alcanza el desarrollo:

"Para crecer necesitamos cada vez más
energía. No podemos darnos el lujo de
desaprovechar recursos en generación
eléctrica" (presidenta Michelle Bachelet,
2008).

"Chile es un país rico en agua. Y el agua es energía limpia y renovable. No produce emisiones contaminantes ni efecto invernadero" (presidente Sebastián Piñera, 2011a). "Necesitamos una energía más segura, para no enfrentar riesgos de apagones. Y más económica, porque hoy tenemos una energía muy cara, con la que sufren las familias y también el sector productor cuando tiene que competir" (presidente Sebastián Piñera, 2011b).

De esta manera, el discurso hegemónico de represas y desarrollo ha sido presentado frente a la sociedad chilena, reforzado por los actores políticos de las dos más grandes coaliciones y fortalecido por los medios de comunicación. Es más, si Chile alcanza el desarrollo podrá en el futuro prescindir de construir represas: "Si la decisión (de construir HidroAysén) tuviese que tomarse en 50 años más, quizá este proyecto se rechazaría. Pero, mirado desde la perspectiva actual, si en 50 años más la sociedad cambiara de parecer y se declarara categóricamente descontenta con esas represas, podrá fácilmente demolerlas y recuperar en gran parte aquellos ecosistemas afectados por aquellas, pues habrá acumulado la riqueza necesaria para reemplazarlas por otras fuentes de energía. (El Mercurio, Editorial, 14 de mayo de 2011). Como he expuesto en este artículo, los efectos de las represas son de carácter irreversible y no dependen del nivel de desarrollo que alcance una nación. 


\section{Cambio en las estructuras de poder}

El caso de HidroAysén ilustra que la construcción de represas es un proceso cultural, político, judicial, social, económico y ambiental que trasciende el contexto inmediato de un proyecto hidroeléctrico determinado. Como proyectos ecológicos-políticos materiales y simbólicos, las represas afectan las estructuras de poder de una sociedad y el medio ambiente que esta habita. El debate sobre represas cuenta con una oposición organizada en una serie de poderosas ONGs internacionales que apoyan ONGs nacionales. En ellas confluyen una serie de actores como intelectuales, académicos, profesionales, políticos, organizaciones locales y artistas, entre otros. En el caso de Chile la organización más grande se llama "Patagonia sin Represas" donde confluyen una serie de coaliciones ciudadanas. Desde Patagonia sin Represas se ha desarrollado una campaña de oposición con carteles en lugares públicos, información a través de los medios de comunicación, y la utilización de las Ilamadas redes sociales. La campaña ha producido publicaciones, libros, documentales, discos y seminarios en varias partes del país.

HidroAysén ha enfrentado la resistencia de estos sectores, pero además, el rechazo de un importante sector de la población chilena en un contexto de movilizaciones sociales que cuestionan la legitimidad del modelo neoliberal chileno. Pero además, se han articulado contradiscursos sobre la Patagonia que están relacionados con la forma en la cual esta ha sido cultural e históricamente construida: la Patagonia es representada como una "Reserva de Vida", parte integral de la identidad territorial de Chile y patrimonio nacional, conformada por paisajes prístinos y sublimes. Se ha movilizado fuertemente una idea de Patagonia desarrollada por el Estado chileno desde los conflictos limítrofes con Argentina, donde el patriotismo, los conflictos por la privatización del agua y la defensa del medio ambiente han sido colocados juntos, constituyendo a la Patagonia como un bien común. De esta manera, el "conflicto HidroAysén" es el resultado de una serie de críticas contra el autoritarismo neoliberal que aun rige a Chile, especialmente visible en el sector eléctrico, y la construcción cultural de la Patagonia (se desarrollará el tema en futuros trabajos).

HidroAysén significó impactos políticos para el gobierno de turno. Según la Encuesta Adimark, la autorización del proyecto hidroeléctrico significó una caída en la aprobación del presidente Piñera desde un $41 \%$ a un 36\% (Encuesta Adimark, mayo de 2011). De esta manera, el conflicto HidroAysén se sumó a una serie de conflictos sociales, ambientales, étnicos y regionales que tuvo que afrontar el gobierno de Piñera y que debilitaron su aprobación popular.

Pero también HidroAysén reforzó la estructura de poder de algunos actores, en referencia al control sobre recursos naturales que poseen, y la inversión que pretenden realizar. Por ejemplo, la empresa Colbún, propiedad de la familia Matte, de gran influencia política y económica en Chile, decidió congelar su participación en HidroAysén:

"Mientras no exista una política nacional
que cuente con amplio consenso y otor-
gue los lineamientos de la matriz energé-
tica que el país requiere (...) Será misión
de los poderes Ejecutivo y Legislativo
consensuar los cambios institucionales y
regulatorios necesarios para viabilizar los
proyectos de generación y transmisión
que demanda el desarrollo económico y
social de Chile" (Larraín Matte, 2012).

De esta manera, el proyecto HidroAysén más que ser solo un proyecto privado de carácter extractivo, pretende transformarse en una transformación de la Política Energética de Chile.

Dicha transformación se basaría en el proyecto del Gobierno de construir una "carretera pública eléctrica", "para permitir a múltiples generadores, también a distribuidoras y consumidores, integrarse al Sistema Interconectado Nacional" (Piñera, 2011a), cuyos objetivos son atender en mejores condiciones la demanda eléctrica, evitar congestiones en el transporte de la energía y reducir los costos de operación, y que hoy aparece presionando políticamente por Colbún, en busca de la creación de los instrumentos legales y la in- 
fraestructura pública que creen las condiciones para desarrollar el proyecto HidroAysén.

\section{Conclusiones}

El presenta artículo ha planteado la existencia de una ecología política sobre represas. Para ello se ha analizado una serie de estudios teóricos y empíricos que sirvan como marco de referencia para entender no solamente los impactos de dichas obras, sino también las condiciones discursivas que existen en su preinstalación. Desde la ecología política, las represas han sido definidas como medioambientes socionaturales que representan el poder de la elite para manejar no solamente el agua sino sistemas sociales complejos. Las represas representan símbolos de progreso y prestigio nacional que necesitan fuertes grados de consenso.

Existe un debate mundial sobre las represas donde toman partes una diversidad de actores. Básicamente las represas son consideradas como una estrategia limpia, barata y eficiente para producir energía, cuyo fin es el crecimiento económico. Dicho discurso es apoyado por una serie de discursos técnicos y políticos sobre la naturaleza, el agua, el medio ambiente y las poblaciones humanas de profundo carácter discriminador y antidemocrático. Existen contradiscursos, principalmente desarrollados por ONGs internacionales que plantean usos alternativos del agua de los ríos, la necesidad de fuentes de energía renovables no convencional y cambios en los hábitos de consumo. Desde estos dos discursos pueden entenderse muchos de los conflictos sociales y ambientales originados por la construcción de represas.

Las grandes represas son generalmente justificadas por sus beneficios macroeconómicos a escala regional y nacional. Sin embargo, sus impactos físicos y simbólicos se concentran localmente, pero también pueden llegar a influir las dinámicas políticas y económicas a escala nacional. En esto radica el entendimiento de los discursos y la articulación de las condiciones para transformaciones ambientales y sociales de carácter material y simbólico. El Análisis Crítico de Discurso permite captar cómo las relaciones entre naturaleza y sociedad están mediadas por una serie de representaciones culturales desde donde se ejerce el poder.

Las represas producirían impactos directos e indirectos sobre el ambiente y la sociedad desde el estado de preconstrucción. Como lo demuestra el caso de HidroAysén, existen consensos no democráticos, abalados en discursos técnicos que impulsan la transformación ambiental y social. Sin embargo, cuando estos consensos se rompen, se abren espacios para la discusión sobre el rol del Estado, la diversificación de la matriz energética chilena, la necesidad de contar con mejores leyes ambientales y una institucionalidad acorde a los nuevos escenarios. HidroAysén ha permitido el debate sobre energía y medio ambiente, y ha posibilitado la visibilidad de la Patagonia aysenina, cuyos problemas socioeconómicos están lejos de ser resuelto por un proyecto hidroeléctrico.

\section{Referencias bibliográficas}

ADAMS, W. Social Impacts of Large Dams Equity and Distributional Issues. Cape Town: World Commission on Dams, 2000.

ALTINBILEK, D. The Role of Dams in Development. Water Science and Technology, 2002, Vol. 45, № 8, p. 169-180.

BACHELET, M. Discurso Presidencial del 21 de Mayo. Santiago de Chile: Segpres, 2008.

BAKKER, K. The Politics of Hydropower: Developing the Mekong. Political Geography, 1999, Vol. 18, p. 209-232.

BAUER, C. Contra la Corriente: Privatización, Mercados de Agua y el Estado en Chile. Santiago de Chile: Lom Ediciones, 2002.

BAUER, C. Dams and Markets: Rivers and Electric Power in Chile. Natural Resources Journal, 2009, Vol. 49, p. 583-651.

BERNSTEIN, S. Sector Eléctrico. Soluciones privadas a problemas públicos. Santiago de Chile: Instituto Libertad y Desarrollo, 1991.

BLAIKIE, P. The Political Economy of Soil Erosion in Developing Countries. Essex: Longman, 1985. 
BLAIKIE, P. \& BROOKFIELD, H. Land Degradation and Society. New York: Methuen \& Co. Ltd., 1987.

BLAIKIE, P. \& SPRINGATE-BAGINSKI, O. Setting Up Key Policy Issues in Participatory Forest Management. In: SPRINGATE-BAGINSKI, P. \& BLAIKIE, O. (editors). Forest, People \& Power: The political ecology of reform in South Asia. London: Earthscan, 2007, p. 1-14.

BRAUN, B. \& CASTREE, N. Remaking Reality: Nature at the Millenium. New York: Routledge Chapman \& Hall, 1998.

BUDDS, J. Power, Water and Neoliberalism: The Political Ecology of Water in Chile. Singapore Journal of Tropical Geography, 2004, Vol. 25, N³, p. 322-342.

BUDDS, J. Contested H2O: Science, Policy and Politics in Water Resources Management in Chile. Geoforum, 2009, Vol. 40, p. 418-430.

CUMMINGS, B. Dam the Rivers; Damn the People: Hydroelectric Development and Resistance in Amazonian Brazil. Geolournal, 1995, Vol. 35, N² 2, p. 151-160.

DORCEY, A. Large Dams: Learning From the Past, Looking At the Future. Workshop Proceedings. Gland: The World Bank, 1997.

ÉGRÉ, D. \& SENÉCAL, P. Social Impact Assessments of Large Dams Throughout the World: Lessons Learned Over Two Decades. Impact Assessment and Project Appraisal, 2003, Vol. 21, N³, p. 215-224.

EL MERCURIO. Editorial. Santiago de Chile: Aguilar ediciones, 2011.

ERAKHTIN, B. Theoretical Basis of Designing Concrete Dams with Consideration of Construction Operation. Hydrotechnical Construction, 1998, Vol. 32, № 2, p. 82-88.

ESCOBAR, A. After Nature: Steps to an Antiessentialist Political Ecology. Current Anthropology, 1999, Vol. 40, № 1, p. 1-30.

FAIRCLOUGH, N. Critical Discourse Analysis: the Critical Study of Language. New York: Longman Publishing, 1995.
FERNÁNDEZ, D. (2011). Entrevista con Jorge Navarrete.

Disponible en Internet: http://www.youtube.com/watch? $v=Z 4 d S D J T 97 s A$

FORSYTH, T. Critical Political Ecology: The politics of environmental science. New York: Routledge, 2003.

HARVEY, D. Justice, Nature and the Geography of Difference. Oxford: Blackwell, 1996.

HEMING, L.; WALEY, P. \& REES, P. Reservoir Resettlement in China: Past Experience and the Three Gorges Dam. The Geographical Journal, 2001, Vol. 167, №3, p. 195-212.

HOWITT, R. Rethinking Resource Managment. London: Routledge, 2001.

JAPAN COMMISSION ON LARGE DAMS (JCLD). EADC Papers. London: Taylor \& Francis Group, 2009.

JORGENSEN, M. \& PHILLIPS, L. Discourse Analysis as Theory and Method. London: SAGE, 2002.

KAIKA, M. Dams as Symbols of Modernization: The Urbanization of Nature Between Geographical Imagination and Materiality. Annals of the Association of American Geographers, 2006, Vol. 96, №2, p. 276-301.

LARRAÍN MATTE, B. (2012). Carta de Colbún a la Superintendencia de Valores y Seguros, 30 de Mayo.

LEDEC, G. \& QUINTERO, J. Good Dams and Bad Dams: Environmental Criteria for Site Selection of Hydroelectric Projects. New York: Latin America and Caribbean Region Sustainable Development Working Paper 16, 2003.

MARTínEZ-ALIER, J. Political Ecology of Industrial Tree Plantations and Large Dams. Capitalism Nature Socialism, 1998, Vol. 9, No 3, p. 141-146.

McCARTNEY, M. Living with Dams: Managing the Environmental Impacts. Water POlicy, 2009, Vol. 11, № 1, p. 121-139.

MOLLE, F. Irrigation and Water Policies in the Mekong region: Current Discourses and Practices. Colombo: IWMI, 2005. 
MURDOCH, J. Post-Structuralist Geography: A Guide to Relational Space. London: SAGE, 2006.

NÜSSER, M. Political Ecology of Large Dams: a Critical Review. Petermanns Geographische Mitteilungen, 2003, Vol. 147, $\mathrm{N}^{\circ} 1$, p. 20-27.

PEET, R. \& WATTS, M. (editors). Liberation Ecologies: Environment, development, social movements. London: Routledge, 1996.

PEET, R.; ROBBINS, P. \& WATTS, M. (editors). Global Political Ecology. London: Routledge, 2011.

PIÑERA, S. Discurso Presidencial del 21 de Mayo. Santiago de Chile: Segpres, 2011 a.

PIÑERA, S. Piñera e HidroAysén: "Los que se oponen a todo están profundamente equivocados". (2011b). Disponible en Internet:

http://www.emol.com/noticias/nacional/2011/05/11/480918/pinera-e-hidroaysenlos-que-se-oponen-a-todo-estan-profundamente-equivocados.html

PRIETO, M. \& BAUR, C. Hydroelectric Power Generation in Chile: An Institutional Critique of the Neutrality of Market Mechanisms. Water International, 2012, Vol. 37, No 2, p. 131-146.

RODRIGO, P. \& ORREGO, J.P. (editor). Patagonia Chilena iSin Represas! Santiago: Ocho Libros, 2007.

ROSENBERG, D; BODALY, R.D.; \& USHER, P. Environmental and Social Impacts of Large Scale Hydroelectric Development: Who is Listening? Global Environmental Change, 1995, Vol. 5, No 2, p. 127-138.

ROSENBLUT, J. Presidente de Endesa reitera Ilamado a Comité de Ministros para que revise reclamaciones a HidroAysén, 2012.

Dsiponible en Internet: http://www.latercera.com/noticia/negocios/2013/04/655518870 -9-presidente-de-endesa-reiterallamado-a-comite-de-ministros-para-querevise.shtml

SCUDDER, T. The Future of Large Dams. London: Earthscan, 2005.
SMITH, N. Uneven Development: Nature, Capital, and the Production of Space. Oxford: Blackwell, 1984.

SULLIVAN, S. \& STOTT, P. Political ECology: Science, Myth and Power. London: Arnold, 2000.

SWYNGEDOUW, E. Modernity and $\mathrm{Hi}-$ bridity: Nature, Regeneracionismo, and the Production of the Spanish Waterscape, 1890-1930. Annals of the Association of American Geographers, 1999, Vol. 89, № 3, p. 443-465.

SWYNGEDOUW, E. Modernity and the Production of the Spanish Waterscape, 18901930. En Zimmerer, T.; Bassett, K.S. (editors), Political Ecology. An integrative approach to geography and environment-development studies, London: The Guildorf Press, 2003, p. 94-112.

SWYNGEDOUW, E. Technonatural Revolutions: The Scalar Politics of Franco's HydroSocial Dream for Spain, 1939-1975. London: Royal Geographical Society, 2007.

TILT, B.; BRAUN, Y. \& HE, D. Social Impacts of Large Dam Projects: A Comparison of International Case Studies and Implications for Best Practice. Journal of Environmental Management, 2009, Vol. 90, p. 249-S257.

TOKMAN, M. Política Energética: Nuevos Lineamientos. Transformando la Crisis Energética en una Oportunidad Política. Santiago de Chile: CNE, 2008.

VAN DIJK, T. Ideology: A Multidisciplinary Approach. London: SAGE, 1998.

VAN DIJK, T. Principles of Critical Discourse Analysis. London: Sage, 2001.

WOODS, N. Describing Discourses: A Practical Guide to Discourse Analysis. London: Hodder Arnold, 2006.

WORSTER, D. Hydraulic Society in California: An Ecological Interpretation. Agricultural History, 1982, Vol. 56, № 3, p. 503-515. 
